\title{
Centro de material de esterilização: parâmetros espaciais e riscos físicos*
}

\author{
Supply and sterile center: spatial parameters and physical risks \\ Centro de material y esterilización: parámetros espaciales y riesgos físicos
}

Andréa Borges Araruna ${ }^{1}$, Maria Belén Salazar Posso ${ }^{2}$

RESUmo: Objetivo: O objetivo deste trabalho foi identificar os riscos físicos presentes no Centro de Material e Esterilização de dois Estabelecimentos de Assistência à Saúde (EAS) públicos. Método: Trata-se de um estudo descritivo-exploratório, de campo, transversal, com abordagem quantitativa. Por meio de um checklist baseado na RDC ANVISA n ${ }^{\circ}$ 50/2002, coletaram-se os dados da dimensão físicoespacial dos Centros de Material e Esterilização e da presença de riscos físicos, em maio de 2013. Resultados: Na estrutura organizacional, verificou-se que ambos os EAS possuem Centro de Material e Esterilização centralizado; porém, seu dimensionamento físico-espacial e fluxo direcional entre as áreas são inadequados, assim como seus pisos, tetos, paredes, portas, ventilação, temperatura e iluminação não atendem a essa resolução. Conclusão: Ambos os hospitais respeitam parcialmente as normas estabelecidas, comprometendo a qualidade do trabalho desenvolvido no Centro de Material e Esterilização e, ainda, tais condições constituem-se em sério risco para a saúde dos trabalhadores.

PALAVRAS-CHAVE: Riscos ocupacionais; Enfermagem; Esterilização; Arquitetura Hospitalar.

ABSTRACT: Objective: The aim of the present study was to identify physical risks in the Supply and Sterile Center of two public hospitals. Method: This is a field, cross-sectional, descriptive, exploratory study of quantitative approach. Through a checklist based on the resolution RDC No. 50/2002 of the Brazilian Health Surveillance Agency - ANVISA, we collected data of the physical-spatial dimension of the Central Sterile Supply and the presence of physical risks in May 2013. Results: Regarding the organizational structure, we found that both Hospitals have a centralized Central Sterile Supply; however, their physical-spatial dimensioning and directional flow between areas are inadequate, and their floors, ceilings, walls, doors, ventilation, temperature and lighting do not meet the resolution standards. Conclusion: Both hospitals comply with the established standards partially, which compromises the quality of the work developed in the Supply and Sterile Center and jeopardizes the workers' health.

KEYWORDS: Occupational risks; Nursing; Sterilization; Hospital design and construction

RESUMEN: Objetivo: el objetivo fue Identificar los riesgos físicos presentes en el Centro de Material y Esterilización de dos hospitales públicos. Método: Se trata de un estudio descriptivo-exploratorio, de campo, transversal, con abordaje cuantitativo. Los datos fueron recolectados mediante checklist de la RDC ANVISA n ${ }^{\circ}$ 50/2002 en mayo de 2013. Resultados: en la estructura organizacional se pudo verificar que ambos hospitales poseen Centro de Material y Esterilización centralizado; sin embargo, su dimensión y los flujos direccionales entre sus áreas resultan inadecuados, así como sus pisos, techos, paredes, puertas, ventilación, temperatura e iluminación tampoco cumplen la normativa. Conclusión: los dos hospitales respetan las normas establecidas, aunque parcialmente, comprometiendo la calidad del trabajo desarrollado en el Centro de Material y Esterilización, y constituyendo un serio riesgo para la salud de los trabajadores.

PALABRAS CLAVE: Riesgos laborales. Enfermería. Esterilización. Arquitectura de hospitales.

${ }^{1}$ Enfermeira. Mestre em Bioengenharia (UNIVAP). Enfermeira do Hospital Geral de Caxias -MA. Cel: (99) 8193-7933; (99) 8807-9730. E-mail: andreaborges19@hotmail.com ${ }^{2}$ Enfermeira. Doutora (EEUSP). Professor pesquisador do Instituto de Desenvolvimento e Pesquisa da Universidade Vale do Paraíba. Rua Iperoig, 749 apto 111 Perdizes, CEP 05016-000, São Paulo, SP, Brasil. Telefone (12) 3947-1106; (11) 99625-8831. E-mail: mbelen@terra.com.br

*Extraído da dissertação de Mestrado em Bioengenharia, intitulada: Centro de material e esterilização: dimensão físico-espacial e a presença de riscos físicos, apresentada à UNIVAP. Defendida em 4/07/2013. 


\section{Introdução}

O Estabelecimento de Assistência à Saúde (EAS) caracteriza-se por oferecer assistência preventiva e curativa de complexidade variável e de qualidade, em que vários fatores contribuem para o alcance dessa missão $0^{1}$; porém, por outro lado, a alta sofisticação de equipamentos eletromédicos necessários para garantir tal missão pode apresentar uma série de fontes potenciais de riscos ${ }^{2}$. O EAS é composto por várias unidades de assistência, entre as quais o Centro de Material e Esterilização (CME), uma unidade de apoio técnico responsável por processar todo o material a ser utilizado em todos os setores de um EAS $^{3}$.

Nesse contexto, o CME destaca-se por ser uma área do hospital - considerada crítica - que está envolvida diretamente no controle de infecções e que exerce importante papel de proteção anti-infecciosa dentro dos serviços de saúde ${ }^{3}$. Segundo o Ministério da Saúde ${ }^{4}$, o CME é definido como: “... conjunto de elementos destinados à recepção, expurgo, preparo e esterilização e guarda e distribuição do material para unidades de estabelecimentos de saúde". A partir dessa definição, a Agência Nacional de Vigilância Sanitária (ANVISA), pela Resolução de Diretoria Colegiada - RDC $n^{\circ} 50 / 2002^{5}$, regulamentou o planejamento, a programação, a elaboração e a avaliação de projetos físicos de EAS.

No Brasil, a Resolução de Diretoria Colegiada - RDC $n^{0} 50 / 2002^{5}$ - regulamenta o planejamento, a programação, a elaboração e a avaliação de projetos físicos de EAS, ressaltando que todos os projetos desses estabelecimentos deverão ser elaborados de acordo, entre outros, com a referida resolução, a fim de garantir a qualidade dos serviços prestados e oferecer estrutura adequada para desenvolvimento das atividades laborais dos profissionais.

O interesse em avaliar as estruturas dos CME dos EAS de um município do Estado do Maranhão deveu-se à amplitude do trabalho que ali é realizado. Assim, questionou-se: 'As estruturas físicas dos CME obedecem à legislação vigente para esse fim?'; 'Essa estrutura apresenta agentes físicos capazes de interferir na saúde ocupacional dos profissionais que ali desempenham suas atividades?'; 'Por conseguinte, quais seriam os riscos físicos a que estão expostos esses trabalhadores?'.

Os EAS são um ambiente em que se desenvolvem atividades múltiplas e complexas, possuindo um universo tecnológico sofisticado para melhor assistir a seus clientes; tal universo, por outro lado, apresenta fontes potenciais de riscos, cujos agentes biológicos, físicos, químicos, psicológicos e ergonômicos podem ser determinantes de acidentes e/ou doenças ocupacionais $^{6-8}$. Assim, a Portaria MET 3.214/78 do Ministério do Trabalho e Emprego (MET) ${ }^{9}$ classifica os riscos em:

Riscos Físicos: calor, frio, ruído, vibrações, pressões anormais, radiações ionizantes e não ionizantes, umidade, sendo todos bem descritos nas Normas Regulamentadoras 9 e 15 (NR-09 e NR-15 $5^{9-12}$;
Riscos Químicos: substâncias, compostos ou produtos que possam invadir o organismo, seja pela via respiratória - nas formas de poeiras, fumos, névoas, neblinas, gases ou vapores - ou que, devido à natureza da exposição, possam ter contato com a pele ou serem absorvidos por ingestão; esses agentes são caracterizados e avaliados qualiquantitativamente $^{9-13}\left(\mathrm{NR}-09\right.$ e NR-15) ${ }^{9-13}$;

Riscos Biológicos: são bactérias, fungos, bacilos, parasitas, protozoários, vírus, entre outros ${ }^{9-13}$;

Riscos Ergonômicos: fatores físicos e organizacionais que afetam o conforto da atividade profissional e, consequentemente, as características psicofisiológicas do trabalhador ${ }^{9-11,13}$.

Esses riscos podem, ainda, ser classificados como primários, secundários e terciários ${ }^{14}$, cuja potencialidade de agravo ao trabalhador dependerá do cumprimento da Legislação pertinente e do conhecimento e da atenção do profissional no desenvolver de suas atividades, incluindo os limites de tolerância. Acrescente-se a tais riscos o tipo de manutenção, seja este advindo da instalação elétrica, dos equipamentos gerais e eletromédicos, de incêndio/explosão, do arranjo físico inadequado, do tipo de armazenamento de material, da inadequação do equipamento de apoio, do espaço restrito para a movimentação de equipamentos e pessoal, entre outros ${ }^{15}$.

Dentro desse ambiente assistencial, o CME, pelas características do trabalho ali desenvolvido, é considerado propício à insalubridade dos profissionais que ali desempenham suas atividades ${ }^{6}$; porém, ao mesmo tempo, é uma unidade de vital importância para as demais unidades hospitalares e tem relevante papel na qualidade da assistência prestada aos clientes e na segurança dos profissionais que a desempenham. Portanto, é fundamental que suas instalações sejam corretamente planejadas.

\section{Objetivo}

Caracterizar os parâmetros físicos e as possíveis fontes de risco dos CME de dois EAS de um município do Estado do Maranhão, em conformidade com a RDC 50/20025.

\section{Método}

A pesquisa enquadra-se como pesquisa descritivoexploratória, transversal, de campo, com abordagem quantitativa, realizada no CME de EAS públicos de um município do Estado do Maranhão, mediante observação direta, utilizando-se um checklist adaptado de Guadagnin 2006 e elaborado conforme os requisitos da RDC ANVISA $\mathrm{N}^{\circ} 50 / 2002^{5}$.

Foram dois (100\%) os EAS públicos, sendo que um deles presta assistência materno-infantil denominado "EAS A" e o outro, atendimento geral e especializado, denominado "EAS B", que possuíam CME. O EAS A é de médio porte, porém é referência para 15 municípios no perímetro regional e 
47 municípios no perímetro macrorregional de um município do Estado do Maranhão. O EAS A atende uma média de 450 procedimentos obstétricos por mês, possui 50 leitos obstétricos em ALCON (alojamento conjunto), dez leitos de pré-parto, duas salas de parto normal, duas salas cirúrgicas, dois leitos de Recuperação Pós-anestésica; também há 11 leitos de Unidade de Terapia Intensiva (UTI) neonatal, cinco leitos de berçário, um CME, um banco de leite humano e uma sala de transfusão.

O EAS B é de médio porte, com 125 leitos, que presta atendimento de média e alta complexidade aos municípios integrados na macrorregião de um município maranhense, oferecendo atendimento a diversas especialidades. Possui setor de emergência, composta de Recepção, Consultório, Sala de parada cardiorrespiratória, Sala de raios-X, CME, Farmácia, Centro Cirúrgico (CC), UTI, unidades de internação divididas em quatro alas, além de lavanderia, Nutrição, Laboratório, setores administrativos, Serviço Social e Comissão de Controle de Infecção Hospitalar (CCIH).

Os dados foram coletados após anuência assinada pela Direção dos EAS A e B, autorizando o acesso ao espaço físico para a realização da pesquisa, pelo fato de o estudo não envolver seres humanos, no período 15 a 30 de maio de 2013. Dessa forma, o próprio pesquisador, após a autorização dos gestores de cada instituição, adentrou a unidade de CME das instituições.

A coleta de dados se deu por meio de um check list adaptado de Guadagnin $2006^{16}$ e elaborado conforme o preconizado pela RDC ANVISA $n^{\circ} 50 / 2002^{5}$, composto de três partes: Parte A- Identificação dos EAS, composta de quatro itens: Nome do EAS, Finalidade, Porte e Número de Salas Cirúrgicas; Parte B- Identificação do CME, abrangendo três itens: Tipo de estrutura organizacional do CME (centralizado, semicentralizado ou descentralizado); Área do CME; Setores presentes na CME: Recepção, Lavagem, Preparo e Acondicionamento, Esterilização, Armazenamento e Distribuição dos Artigos, Obediência ao fluxo correto dos artigos; Parte C: Padrões arquitetônicos específicos de cada setor: Expurgo, Preparo e Acondicionamento; Esterilização física e química, e Armazenamento e Distribuição de Material, abrangendo tipos e conservação de bancadas, piso, paredes, tetos, cor, janelas, portas, ventilação e climatização, e presença de exaustores, iluminação; instalação elétrica; temperatura; nível de pressão sonora; presença de objetos móveis (foco auxiliar com base instável e altura regulável ou fixa); objetos suspensos nos tetos e fixos e salientes nas paredes (altura do piso); ralos com abertura sobressalente no piso; tipo de recipiente para esterilização química; presença de escada de dois degraus (material e conservação).

Para a mensuração da área total e de cada setor, foi utilizado o metro, e para registro icônico dos detalhes de construção, utilizou-se uma câmera iSight ${ }^{\circledR}$ de cinco megapixels da Apple ${ }^{\circledR}$. Os resultados obtidos foram armazenados em planilha do programa do MICROSOFT OFFICE EXCEL 2007®, sendo suas frequências absolutas e percentuais analisadas descritiva e quantitativamente, $\mathrm{e}$ apresentadas sob forma de Tabelas e Quadro.

\section{Resultados}

Os resultados da pesquisa são expostos e discutidos em forma de Tabelas e Quadro, conforme os parâmetros da RDC $\mathrm{n}^{\mathrm{o}}$ 50/2002 ${ }^{5}$ e Guadagnin $2006^{16}$, além de Posso; Sant'Anna ${ }^{8}$, Bokor $^{12}$, para citar alguns que diretamente se reportaram aos riscos potenciais presentes na estrutura física dos $\mathrm{CME}$ estudados.

A Tabela 1 apresenta a caracterização dos dois EAS pesquisados, quanto a porte (número de leitos), nível de complexidade, tipo de atendimento e referência de atendimento em que ambos os EAS são de médio porte, porém diferentes no tipo de atendimento.

Pela Tabela 2, detecta-se que ambos os EAS (100\%) apresentam a tinta acrílica como revestimento da parede, e as portas de madeira, revestidas com tinta de esmalte sintético. Quanto ao piso, há realidades diferentes: em todo o CME do EAS A, o piso é de granilite (50\%), e em todo o EAS $\mathrm{B}$, o piso é de granito (50\%); por fim, ocorre a presença de revestimento do teto, do tipo forro contínuo, em ambos os EAS (100\%); porém, encontrou-se forro removível somente no EAS B (50\%).

O Quadro 1 mostra a presença das fontes de risco físico nos CME de ambos os EAS estudados, salientando a integridade do piso e o teto contínuo no EAS 1.

Tabela 1. Caracterização dos Estabelecimentos de Assistência à Saúde (EAS) quanto a número de leitos, nível de complexidade, tipo de atendimento. Maranhão, 2013.

\begin{tabular}{lcc}
\hline \multicolumn{1}{c}{ Características } & EAS A & EAS B \\
\hline Número de leitos & 78 & 125 \\
Nível de complexidade & Alta & Alta \\
Tipo de atendimento & Urgência/Ambulatorial/ & Urgência/Ambulatorial/ \\
Referência de atendimento & Internação/SADT* & Internação/SADT*/Vigilância em Saúde \\
\hline
\end{tabular}

Fonte: Cadastro Nacional de Estabelecimentos de Saúde, 2013. *Serviço de Apoio à Diagnose e Terapia. 
Tabela 2. Tipos de revestimento em piso, paredes, portas e teto dos CME dos EAS. Maranhão, 2013.

\begin{tabular}{|c|c|c|c|c|c|c|}
\hline \multirow{2}{*}{ Tipos } & \multicolumn{2}{|c|}{ EAS A } & \multicolumn{2}{|c|}{ EAS B } & \multicolumn{2}{|c|}{ TOTAL } \\
\hline & $\mathbf{N}$ & $\%$ & $\mathbf{N}$ & $\%$ & $\mathbf{N}$ & $\%$ \\
\hline Granilite (piso) & 1 & 50 & 0 & 0 & 1 & 50 \\
\hline Granito (piso) & 0 & 0 & 1 & 50 & 1 & 50 \\
\hline Tinta acrílica na parede & 1 & 50 & 1 & 50 & 2 & 100 \\
\hline Tinta esmalte sintético em portas/madeira & 1 & 50 & 1 & 50 & 2 & 100 \\
\hline Forro contínuo no teto (gesso) & 1 & 50 & 1 & 50 & 2 & 100 \\
\hline Forro removível no teto (PVC) & 0 & 0 & 1 & 50 & 1 & 50 \\
\hline
\end{tabular}

Fonte: Pesquisa direta, 2013.

Quadro 1. Presença das fontes de risco nos CME dos EAS. Maranhão, 2013.

\begin{tabular}{|c|c|c|}
\hline Fontes de risco físico & EAS A & EAS B \\
\hline Presença de porta de madeira com ranhuras & Sim & Sim \\
\hline Ausência de exaustor (expurgo+ área de esterilização física) & $\operatorname{Sim}$ & $\operatorname{Sim}$ \\
\hline Ausência de janelas (expurgo) & Sim & Sim \\
\hline Esterilização física compartilhada com a área de preparo & Sim & $\operatorname{Sim}$ \\
\hline Dimensionamento incorreto & Sim & $\operatorname{Sim}$ \\
\hline Pisos danificados & Não & $\operatorname{Sim}$ \\
\hline Tetos falsos e removíveis & Não & $\operatorname{Sim}$ \\
\hline Ausência de ar condicionado & Sim & $\operatorname{Sim}$ \\
\hline
\end{tabular}

\section{Discussão}

No que se refere ao número de leitos ofertados (Tabela 1), sabe-se que estes determinarão vários critérios no planejamento de um CME, como o dimensionamento das áreas específicas e a sua área total. Na estrutura organizacional, verificou-se que ambos os EAS possuem CME centralizado. Considera-se esta característica importante para a qualidade do processamento dos artigos, tendo em vista que a centralização permite o emprego de pessoal especialmente habilitado, com supervisão do trabalho, bem como a padronização de técnicas de limpeza e distribuição segura dos artigos estéreis ${ }^{3}$.

Pesquisa realizada em 44 hospitais de municípios do Estado de Goiás encontrou uma realidade preocupante, na qual apenas dez hospitais apresentavam CME centralizado, o que dificulta a operacionalização dos serviços, interferindo na qualidade do processamento dos artigos ${ }^{16}$. A qualidade do serviço prestado é infinitamente maior quando as instituições adotam o modelo de CME centralizado, especialmente devido à redução dos riscos de infecção cruzada. Esta recomendação justifica-se mediante três pontos-chave: a eficiência, a economia e a maior segurança para a equipe e para os clientes ${ }^{3}$.

Quanto à área total construída dos CME investigados, em que se avaliou a obediência ao padrão proposto pela $\mathrm{RDC} \mathrm{n}^{0} 50 / 2002^{5}$, proporcionalmente à quantidade de seus leitos, verificou-se que ambos os EAS estão de acordo com o preconizado por essa Resolução. De posse das áreas em metros quadrados de cada setor, faz-se a somatória e, então, se obtém a área total do CME; portanto, a área mínima do EAS A seria de aproximadamente $45,1 \mathrm{~m}^{2}$ e a do EAS B, de $70,54 \mathrm{~m}^{2}$. Observou-se que ambos os hospitais obedecem à $\mathrm{RDC} \mathrm{n}^{\circ} 50 / 2002^{5}$ quanto à área total construída da CME, pois encontrou-se que a área do EAS A foi de $69 \mathrm{~m}^{2}$ e do EAS B de $76 \mathrm{~m}^{2}$.

$\mathrm{O}$ atendimento a esse critério visa à praticidade do trabalho executado; é necessário um espaço amplo para que os profissionais tenham mobilidade para executar suas atividades com segurança técnica e científica - tendo em vista que estas requerem atenção e cuidados -, bem como para o preparo adequado para evitar riscos físicos aos profissionais ${ }^{3}$.

Em relação à existência de setores preconizados pela RDC $\mathrm{n}^{\mathrm{o}} 50 / 2002^{5}$ incluídos no CME, ambos os EAS possuem Recepção, Lavagem, Preparo, Armazenamento e Distribuição para o fluxo correto do processamento dos artigos (recepção, lavagem, preparo, armazenamento e distribuição) e, com isso, evita-se risco para os profissionais. A literatura afirma que área física da CME deve ser projetada de modo que se evite cruzamento de artigos e do fluxo de funcionários ${ }^{3}$.

Nesta variável, avaliaram-se, além do revestimento das paredes e dos pisos, o estado de conservação e a facilidade para traumas mecânicos, caracterizados como riscos físicos aos profissionais; para o alcance desses requisitos, faz-se necessário que as paredes sejam sem trincas, sem saliências e sem rachaduras, com pinturas intactas sem descamações ${ }^{3,5}$. Nos CME observados, verificou-se que, no EAS A, as paredes e os pisos estavam bem conservados, ressaltando que este 
foi construído recentemente. Entretanto, outra realidade foi constatada no EAS B, em que, apesar de as paredes estarem bem conservadas, os pisos apresentavam-se trincados e com desníveis em alguns setores.

$\mathrm{ARDC} \mathrm{n}^{0} 50 / 2002^{5}$ recomenda que as áreas críticas devam ser sempre priorizadas e, mesmo nas áreas semicríticas, os materiais de acabamento devem ser monolíticos, ou seja, lisos, com o menor número possível de ranhuras ou frestas, mesmo que após o uso e a limpeza frequentes, isso os possa tornar escorregadios ${ }^{8}$.

Rochas ígneas, como o granito, têm pouca porosidade e permeabilidade, com uma variabilidade de absorção de água de $0 \%$ a $0,5 \%$ se íntegros; este índice sofre alteração se for danificado pelo intemperismo ${ }^{17}$, desfavorecendo, então, os achados correspondentes para o EAS B. Quanto às portas, cuja abertura se dá para dentro, estas possuem revestimento de esmalte sintético lavável (Tabela 2); no entanto, observouse a presença de saliências e rachaduras, apresentando, assim, riscos físicos aos profissionais.

Quanto ao revestimento do teto (Tabela 2), o EAS A apresenta-o contínuo em toda a sua extensão, sendo que é este o indicado, uma vez que se realiza a vedação do meio externo com o meio interno; também não deve conter saliências e nem frestas que possam dificultar a limpeza do ambiente ${ }^{5}$. O teto do EAS B, por sua vez, possui revestimento contínuo em toda CME, exceto na área de distribuição, em que é removível, do tipo PVC. Convém salientar que nessa área ficam armazenados os artigos já esterilizados, necessitando um ambiente com bom arejamento e limpo; portanto, essa condição é preocupante. De acordo com a literatura, esse tipo de revestimento pode interferir na assepsia não só dos ambientes como do acondicionamento dos materiais, pois existe a possibilidade da passagem de partículas (poeira) por entre as frestas, prejudicando assim as atividades desenvolvidas no setor ${ }^{8}$.

Em relação à área da esterilização física, em ambos os EAS, esta é compartilhada com as áreas de preparo. Há existência de autoclave de barreira apenas no EAS B. Ainda, verificou-se a inexistência de exaustor em ambos os EAS, comprovando, assim, altas temperaturas no ambiente de trabalho, visto que o processo de esterilização física gera calor.

Acrescente-se que o EAS B possui três autoclaves de barreira; destas, duas encontram-se dentro do CME em área específica, porém a distância entre essas autoclaves é inferior a $100 \mathrm{~cm}$, e a outra se encontra na área de preparo; portanto, não se atende a todos os requisitos preconizados para as instalações da área de esterilização física ${ }^{5}$. Lôbo ${ }^{18}$ afirma que, na existência de autoclave de barreira, deverá ser reservada uma área imediata à retirada do material, com uma pequena distância para o setor de armazenagem, de modo que haja uma climatização intermediária entre a saída do instrumental e sua guarda, com exaustão no ambiente, para que a transição térmica evite a condensação, prejudicial à garantia de um material esterilizado. Conforme constatação in loco, o EAS
B possui a referida área, porém não atende corretamente aos critérios exigidos de ventilação e exaustão.

No caso do EAS A, que não possui autoclaves de barreira, faz-se necessária a existência de uma área de manutenção, cuja finalidade é evitar que técnicos em manutenção adentrem no recinto do ambiente limpo ou estéril ${ }^{18}$. Referente à existência de exaustores, o EAS A não os possui e os do EAS B estão com defeito, ocorrendo, desta forma, o comprometimento da esterilidade dos materiais e a criação de um ambiente desfavorável ao trabalho executado pelos profissionais.

No tocante à ventilação, ocorre o comprometimento do CME como um todo e de alguns setores específicos, como o expurgo, a esterilização física e química, e a área de armazenamento, que necessitam de aeração e climatização; porém, isso não ocorrendo em ambos os EAS, percebe-se que suas inexistências afetam tanto o risco laboral quanto a qualidade do processamento dos artigos. Atribuem-se, como causas da Síndrome do Edifício Doente (SED), entre outras, o controle deficiente da temperatura e as modificações inadequadas após construções e/ou manutenção deficientes do sistema de climatização, resultando em sintomas de doenças e mal-estar dos trabalhadores ${ }^{19}$.

Assim, frequentemente, os profissionais de saúde queixam-se de doenças, principalmente as que atingem o trato respiratório sem uma causa definida, podendo estas ser atribuídas ao sistema de ventilação, quando sua manutenção é inadequada ${ }^{19}$. É de se notar como as temperaturas elevadas afetam os profissionais, gerando desconforto, alterações fisiológicas, comprometendo a saúde do trabalhador e seu desempenho profissional devido aos sinais e sintomas que pode apresentar, como: fadiga, erros de percepção e raciocínio, cefaleia, estresses, desencadeando esgotamentos e prostrações ${ }^{3,20}$. Vale salientar que ocorre a predominância de um clima quente no Estado do Maranhão e um ambiente de trabalho com temperaturas elevadas, aliadas ao reduzido espaço de trabalho, certamente transforma o setor em um ambiente insalubre e estressante, comprometendo, também, a produtividade.

Pelo exposto, verifica-se pelo Quadro 1, a presença de inúmeras fontes de risco nos CME dos EAS estudados, que devem ser corrigidos ou minimizados, seguindo o que é determinado pela Legislação vigente e pela literatura especializada nessa Unidade tão essencial à dinâmica hospitalar.

\section{Conclusão}

Conclui-se que ambos os hospitais atendem parcialmente às normas estabelecidas, ocorrendo o descumprimento de requisitos, o que prejudica a qualidade do processamento de artigos hospitalares e constitui fator de risco para a saúde dos trabalhadores.

Assim, as irregularidades encontradas trazem à luz a necessidade da correta disposição da estrutura física da CME, 
face aos riscos existentes pela não obediência aos padrões e normas que a Legislação preconiza para os Estabelecimentos de Assistência à Saúde.

Assim, espera-se que o presente estudo sensibilize os gestores locais para que atentem para a importância de haver a adequação da estrutura física à Legislação vigente e, dessa forma, propiciem ao setor condições favoráveis para o desenvolvimento de suas atividades em sua inteireza, ou seja, oferecer um ambiente de trabalho saudável e seguro aos trabalhadores.

\section{Referências}

1. Silva A. Organização do Centro de material e esterilização In: Graziano KU, Silva A, Psaltikkidis EM, organizadores. Enfermagem em Centro de Material e Esterilização. Barueri: Manole; 2011. p. 1-21.

2. Baptista PCP, Silva A, Bianchi ERF. A saúde do trabalhador de enfermagem no Centro de material e esterilização. In: Graziano KU, Silva A, Psaltikkidis EM, organizadores. Enfermagem em Centro de Material e Esterilização. Barueri: Manole; 2011. p. 301-318.

3. Sociedade Brasileira de Enfermeiros de Centro Cirúrgico Recuperação Anestésica e Centro de Material e Esterilização - SOBECC. Práticas recomendadas da SOBECC. 5. ed. São Paulo: SOBECC; 2013.

4. Brasil. Ministério da Saúde. Agência Nacional de Vigilância Sanitária. Consulta Pública no 34, de 3 de junho de 2009. Dispõe sobre o funcionamento de serviços que realizam o processamento de produtos para a saúde e dá outras providências [internet]. Diário Oficial da União; Brasília; jun. 2009 [acesso em 2013 abr 13]. http://www.anvisa.gov.br/divulga/consulta/index.htm.

5. Brasil. Ministério da Saúde. Resolução RDC n $\mathrm{n}^{\circ}$ 50, de 21 de fevereiro de 2002. Dispõe sobre o regulamento para o planejamento, elaboração, avaliação e aprovação de projetos físicos de Estabelecimentos Assistenciais de Saúde [internet]. Diário Oficial da União; Brasília; 2002. [acesso em 2013 jan 13] Disponível em: http://bvsms.saude.gov.br/bvs/saudelegis/ anvisa/2002/res0050_21_02_2002.html.

6. Mauro MYC, Muzi CD, Guimarães RM, Mauro CCC. Riscos ocupacionais em saúde. R Enferm. UERJ. 2004;12(2):338-45.

7. Brasil. Ministério da Saúde. Agência Nacional de Vigilância Sanitária. Serviços odontológicos: prevenção e controle de riscos [internet]. Brasilia; 2006 [acesso em abr 12]. Disponível em: http:// www.anvisa.gov.br/servicosaude/manuais/manual_odonto.pdf.

8. Posso MBS, Sant'Anna ALGG. Riscos físicos e químicos que envolvem o trabalho em centro cirúrgico. In: Carvalho R, Bianchi
ERF. Enfermagem em centro cirúrgico e recuperação. Barueri: Manole; 2007. p. 335-68.

9. Campanhole A, Campanhole HL. Consolidação das Leis do Trabalho. 108.ed, São Paulo: Atlas; 2004..

10. Brasil. Ministério da Saúde. Portaria $n^{0} 777$, de 28 de abril de 2004. Dispõe sobre os procedimentos técnicos para a notificação compulsória de agravos à saúde do trabalhador em rede de serviços sentinela específica, no Sistema Único de Saúde - SUS. Diário Oficial da União; Brasília; abr. 2004.

11. Brasil. Ministério do Trabalho e Emprego. Portaria $n^{\circ} 1748$, de 30 de agosto de 2011 [internet]. Diário Oficial da União; Brasília; ago. 2011 [acesso em 2013 abr 12]. Disponível em: http://portal. mte.gov.br/data/files/8A7C816A31F92E65013224E36698767F /p_20110830_1748\%20.pdf.

12. Bokor AFK, Carvalho, R. Riscos físicos no centro cirúrgico. Rev. SOBECC. 2004;9(3):20-4.

13. Costa TF, Felli VEA. Exposição dos trabalhadores de enfermagem às cargas químicas em um hospital público universitário da cidade de São Paulo. Rev. Latino-am. Enferm. 2005;13(4):501-8. http:// dx.doi.org/10.1590/S0104-11692005000400007

14. Costa MAF, Costa MFB, Melo NSFO. Biossegurança: ambientes hospitalares e odontológicos. São Paulo: Santos; 2000.

15. Benedett A, Ferraz L, Posso MBS. Os riscos de acidentes de trabalho em unidades de terapia intensiva neonatal. In: Tondin CF, organizador. Contribuições para a humanização do Trabalho. Chapecó: Sinproeste; 2009. p. 236-48.

16. Guadagnin SVT. Avaliação arquitetônica dos centros de material e esterilização de hospitais do interior do estado de Goiás [dissertação]. Goiânia: Faculdade de Enfermagem, Universidade Federal de Goiás; 2006.

17. Soares TN. Revestimento de pisos hospitalares: avaliação das condições de uso em Porto Alegre [monografia]. Porto Alegre: Universidade Federal do Rio Grande do Sul; 2009.

18. Lôbo MCA. A Central de Material Esterilizado Terceirizada e sua arquitetura [monografia]. Salvador: Faculdade de Enfermagem, Universidade Federal da Bahia; 2008.

19. Quadros ME. Qualidade do ar em ambientes internos hospitalares: parâmetros físico-químicos e microbiológicos [tese]. Florianópolis: Faculdade de Enfermagem, Universidade Federal de Santa Catarina, 2008.

20. Castro MES. Condições de trabalho e fatores de risco à saúde dos trabalhadores do centro de material esterilizado do hospital de clínicas da UFPR [tese]. Florianópolis: Faculdade de Enfermagem, Universidade Federal de Santa Catarina; 2003. 